\title{
Symptoms and signs of conjunctivitis as predictors of disease course in COVID-19 syndrome
}

\author{
Martina Ranzenigo ${ }^{1}$, Elena Bruzzesi ${ }^{1}$, Laura Galli ${ }^{1}$, Antonella Castagna ${ }^{1,2}$ and Giulio Ferrari ${ }^{3 *}$ (D)
}

\begin{abstract}
Background: Severe acute respiratory syndrome coronavirus 2 (SARS-CoV-2) can induce conjunctivitis signs and symptoms. However, limited information is available on their impact on COVID-19 disease phenotype.

Quantification of ocular signs/symptoms can provide a rapid, non-invasive proxy for predicting clinical phenotype. Moreover, the existence and entity of conjunctival viral shedding is still debated. This has relevant implications to manage disease spread.

The purpose of this study was to investigate conjunctivitis signs and symptoms and their correlation with clinical parameters, conjunctival viral shedding in patients with COVID-19.

Methods: Fifty-three patients hospitalized between February 25th and September 16th, 2020 at the San Raffaele Hospital, in Milan, Lombardy, Italy with a confirmed diagnosis of SARS-CoV-2 were evaluated. Presence of interstitial pneumonia was confirmed with computed tomography scan imaging. Ocular signs and symptoms, anosmia/ ageusia, clinical/laboratory parameters, and reverse transcriptase-polymerase chain reaction (RT-PCR) from nasopharyngeal and conjunctival swabs for COVID-19 virus were analyzed.
\end{abstract}

Results: Forty-six out of 53 patients showed a positive nasopharyngeal swab for SARS-CoV-2 infection at the time of conjunctival evaluation. All the conjunctival swabs were negative. Conjunctivitis symptoms were present in 37\% of patients. Physician-assessed ocular signs were detected in $28 \%$ of patients.

Patients with ocular symptoms or signs tended to be older: 76.8 years (62.4-83.3) vs 57.2 years (48.1-74.0), $p=0.062$ and had a longer hospitalization: 38 days (18-49) vs. 14 days (11-21), $p=0.005$. Plasma levels of Interleukin- 6 were higher in patients with signs or symptoms in comparison with those without them: $43.5 \mathrm{pg} / \mathrm{ml}$ (19.7-49.4) vs. $8 \mathrm{pg} /$ $\mathrm{ml}(3.6-20.7), p=0.02$. Red cell distribution width was also significantly higher: 15 (14.3-16.7) vs $13.2(12.4-14.4), p=$ 0.001 .

Conclusions: We found that over a third of the patients had ocular signs or symptoms. These had higher prevalence in patients with a more severe infection. No viral shedding was detected in the conjunctiva. Our results suggest that prompt detection of conjunctivitis signs/symptoms can serve as a helpful proxy to predict COVID-19 clinical phenotype.

Keywords: COVID-19, Conjunctivitis, Ocular symptoms

\footnotetext{
* Correspondence: ferrari.giulio@hrr.it

${ }^{3}$ Cornea and Ocular Surface Disease Unit, Eye Repair Lab, IRCCS San Raffaele

Scientific Institute, Via Olgettina, 60, 20132 Milan, Italy

Full list of author information is available at the end of the article
}

\section{Springer Open}

(c) The Author(s). 2021 Open Access This article is licensed under a Creative Commons Attribution 4.0 International License, which permits use, sharing, adaptation, distribution and reproduction in any medium or format, as long as you give appropriate credit to the original author(s) and the source, provide a link to the Creative Commons licence, and indicate if changes were made. The images or other third party material in this article are included in the article's Creative Commons licence, unless indicated otherwise in a credit line to the material. If material is not included in the article's Creative Commons licence and your intended use is not permitted by statutory regulation or exceeds the permitted use, you will need to obtain permission directly from the copyright holder. To view a copy of this licence, visit http://creativecommons.org/licenses/by/4.0/. 


\section{Background}

Conjunctivitis is probably the most common [1]ocular manifestation of COVID syndrome, and specific signs and symptoms have been frequently reported [2-4]. However, it is still unclear whether ocular involvement is associated with viral shedding in the conjunctiva/tears or it is rather a secondary involvement induced by the systemic infection. In this study, we investigated the prevalence of patient-reported ocular symptoms, physician-detected ocular signs, together with anosmia/ ageusia and conjunctival viral shedding in a cohort of patients affected by COVID-19 infection.

\section{Materials and methods}

We evaluated 53 patients hospitalized between February 25th and September 16th, 2020 at the San Raffaele Hospital, in Milan, Italy with a confirmed diagnosis of COVID-19 infection. Presence of interstitial pneumonia was confirmed in all 53 patients with computed tomography scan imaging [5]. This study did not include critically ill (i.e. intubated) patients. Paired (within 3 days) nasopharyngeal and conjunctival swabs were performed during hospitalization. The conjunctival samples were collected from both eyes using a single swab, which was then stored in a vial and analysed within $24 \mathrm{~h}$. Swab specimens were processed by Cobas ${ }^{\circ}$ SARS-CoV-2 Test (Roche), which detects ORF-1a/b and $\mathrm{E}$ gene regions on SARS-CoV-2 genome, designed to be used on the automated Cobas ${ }^{\circ} 6800$ Systems [6]. We also reported the cycle threshold $(\mathrm{Ct})$ values, when available, which are a useful proxy of viral load [7].

A 4-item questionnaire was administered to patients in order to investigate symptoms of conjunctivitis (red eyes, sticky eyes, tearing, burning) and presence of ageusia or anosmia. Finally, patients were evaluated by a study physician for the presence of the following ocular signs: conjunctival hyperaemia, secretion, chemosis and epiphora; previous history of ocular diseases was also collected.

The study was conducted in accordance with the Declaration of Helsinki [8] and the evaluated patients are part of the COVID-19 institutional clinical-biological cohort (Covid-BioB registered on the ClinicalTrials.gov website: NCT04318366), whose study protocol was approved by the Hospital Ethics Committee (protocol number 34/int/2020). Informed consent was obtained according to the Ethic Committee guidelines. Patients' demographic and clinical characteristics, treatment, need/mode of oxygen support as well as values of laboratory parameters during hospitalization were extracted from the Covid-BioB database.

\section{Statistical analysis}

Median values and quartiles (IQR), were used to describe continuous variables while frequencies and percentages were used for categorical variables. Prevalence of patients with ocular symptoms or signs or ageusia or anosmia in the overall sample were estimated with the corresponding 95\% confidence intervals (95\% CIs) using the modified Wald method [9]. Characteristics of patients with or without symptoms or signs were compared using the Chi-square or Fisher's exact test for categorical variables, and the Wilcoxon rank sum test for continuous variables.

Multivariate general linear regression models were fit to estimate mean differences in continuous outcomes (laboratory parameters at conjunctival swab) comparing patients with vs without ocular symptoms or signs, adjusted for two potential confounders [use of steroids before or at ocular swab (yes vs no) and timing of ocular swab execution since hospitalization (continuous variable in days)].

Two-tailed $P$ values are reported and a $P<0.05$ considered to indicate statistical significance.

Statistical analyses were performed with the SAS Software, release 9.4 (SAS Institute, Cary, NC).

\section{Results}

Forty-six out of 53 patients showed a positive nasopharyngeal swab for SARS-CoV-2 infection at the time of conjunctival evaluation and were considered for further analysis. The median age was 65.0 years (48.1-78.0) and $24(52 \%)$ were males. Four patients had a history of previous ocular diseases: 2 patients reported dry eye disease, 1 glaucoma and 1 ectropion.

All the conjunctival swabs were negative. Ocular symptoms were present in 17 [37\%, 95\% confidence interval $(95 \% \mathrm{CI}): 24.5-51.4]$ patients. Red eye was reported by $3(7 \%)$ patients, sticky eyes by $4(10 \%)$, tearing by $6(15 \%)$, burning by $7(16 \%)$. Patients' characteristics according to the presence of ocular symptoms are reported in Table 1.

The study physician observed ocular signs in 13 [28\%, 95\% confidence interval (95\%CI): 17.2-42.7] patients: epiphora in $9(20 \%)$ patients, secretion in $6(13 \%)$, conjunctival hyperaemia in $3(7 \%)$, chemosis in $0 \%$; anosmia was reported in 11 (24\%, 95\% confidence interval (95\% CI): $13.8-38.1]$ patients and ageusia in 13 [28\%, 95\% confidence interval (95\%CI): 17.2-42.7].

Patients with ocular symptoms or signs tended to be older: 76.8 years $(62.4-83.3)$ vs 57.2 years (48.1-74.0), $p=0.062$ and had a longer hospitalization: 38 days (1849 ) vs. 14 days (11-21), $p=0.005$ (Table 1 ).

Importantly, at swab sampling, Ct values were similar between patients with signs or symptoms than those without them [29.5 (24.3-32.8) vs $32.5(25.1-35.0), p=$ 0.432].

Plasma concentrations of Interleukin-6 were significantly increased in patients with signs or symptoms in comparison with those without them: $43.5 \mathrm{pg} / \mathrm{ml}$ (19.7- 
Table 1 Patients' characteristics according to the presence of ocular symptoms

\begin{tabular}{|c|c|c|c|c|}
\hline Variable & $\begin{array}{l}\text { Overall } \\
(n=46)\end{array}$ & $\begin{array}{l}\text { With ocular symptoms or } \\
\text { signs } \\
(n=17)\end{array}$ & $\begin{array}{l}\text { Without ocular symptoms or } \\
\text { signs } \\
(n=29)\end{array}$ & $\begin{array}{l}p- \\
\text { value }^{\S}\end{array}$ \\
\hline \multicolumn{5}{|l|}{ Demographic and clinical characteristics } \\
\hline Age (years) & $65.0(48.1-78.0)$ & $76.8(62.4-83.3)$ & $57.2(48.1-74.0)$ & 0.062 \\
\hline Male gender & $24(52.2 \%)$ & $10(58.8 \%)$ & $14(48.3 \%)$ & 0.552 \\
\hline Days of symptoms before hospitalization & $6(3-11)$ & $6(2.5-10.5)$ & $6(4-11)$ & 0.858 \\
\hline Days to conjunctival swab since hospitalization & $4(1-15)$ & $11(4-22)$ & $3(1-6)$ & 0.023 \\
\hline Days of hospitalization & $18(12-38)$ & $38(18-49)$ & $14(11-21)$ & 0.005 \\
\hline Reported hyperemia & $3(7 \%)$ & $3(21.4 \%)$ & $0(0 \%)$ & - \\
\hline Sticky eyes & $4(10 \%)$ & $4(28.6 \%)$ & $0(0 \%)$ & - \\
\hline Reported tearing & $6(15 \%)$ & $6(42.9 \%)$ & $0(0 \%)$ & - \\
\hline Burning & $7(16.3 \%)$ & $7(50 \%)$ & $0(0 \%)$ & - \\
\hline Hyperemia & $3(7 \%)$ & $3(17.6 \%)$ & $0(0 \%)$ & - \\
\hline Tearing & $9(19.6 \%)$ & $9(52.9 \%)$ & $0(0 \%)$ & - \\
\hline Secretion & $6(13 \%)$ & $6(35.3 \%)$ & $0(0 \%)$ & - \\
\hline Reported chemosis & $0(0 \%)$ & $0(0 \%)$ & $0(0 \%)$ & - \\
\hline Reported anosmia & $11(23.9 \%)$ & $4(23.5 \%)$ & $7(24.1 \%)$ & 0.999 \\
\hline Reported ageusia & $13(28.3 \%)$ & $3(17.6 \%)$ & $10(34.5 \%)$ & 0.315 \\
\hline Antiviral therapy ( $\geq 1$ drug) & $23(50 \%)$ & $11(64.7 \%)$ & $12(41.4 \%)$ & 0.221 \\
\hline Hydroxychloroquine & $10(21.7 \%)$ & $6(35.3 \%)$ & $4(13.8 \%)$ & 0.139 \\
\hline Lopinavir & $1(2.2 \%)$ & $1(5.9 \%)$ & $0(0 \%)$ & 0.370 \\
\hline Remdesivir & $16(34.8 \%)$ & $7(41.2 \%)$ & $9(31 \%)$ & 0.534 \\
\hline Azithromycin & $3(6.5 \%)$ & $2(11.8 \%)$ & $1(3.4 \%)$ & 0.545 \\
\hline Corticosteroids & $22(47.8 \%)$ & $8(47.1 \%)$ & $14(48.3 \%)$ & 0.999 \\
\hline Dexamethasone & $12(26.7 \%)$ & $3(17.6 \%)$ & $9(32.1 \%)$ & 0.488 \\
\hline Methylprednisolone & $9(20 \%)$ & $5(29.4 \%)$ & $4(14.3 \%)$ & 0.265 \\
\hline Prednisolone & $1(2.2 \%)$ & $0(0 \%)$ & $1(3.6 \%)$ & 0.999 \\
\hline CPAP & $6(13 \%)$ & $1(5.9 \%)$ & $5(17.2 \%)$ & 0.390 \\
\hline Venturi mask & $19(41.3 \%)$ & $6(35.3 \%)$ & $13(44.8 \%)$ & 0.555 \\
\hline Glasses & $12(26.1 \%)$ & $6(35.3 \%)$ & $6(20.7 \%)$ & 0.314 \\
\hline Use of CPAPNenturi mask/glasses & $29(63.0 \%)$ & $11(64.7 \%)$ & $18(62.1 \%)$ & 0.998 \\
\hline Eyepiece diseases & $4(8.7 \%)$ & $2(11.8 \%)$ & $2(6.9 \%)$ & 0.619 \\
\hline \multicolumn{5}{|l|}{ Laboratory parameters } \\
\hline Ferritine at hospitalization (ng/mL) & $534(359-1209.5)$ & $495(158-1966)$ & $573(363-1106)$ & 0.999 \\
\hline Ferritine at conjunctival swab (ng/mL) & $390(252-663)$ & $663(158-714)$ & $385(287-520.5)$ & 0.958 \\
\hline Fibrinogen at hospitalization (mg/dL) & $511(447-602)$ & $496.5(432-698)$ & $512(457-598)$ & 0.983 \\
\hline Fibrinogen at conjunctival swab (mg/dL) & $469(415-578)$ & $494(432-592)$ & $461.5(410-544)$ & 0.615 \\
\hline Interleukin-6 at hospitalization (pg/mL) & $21.7(9.8-32.3)$ & $16.2(9.9-51.4)$ & $22.25(6.4-31.5)$ & 0.412 \\
\hline Interleukin-6 at conjunctival swab (pg/mL) & $14.9(5.4-40.6)$ & $43.5(19.7-49.4)$ & $8(3.6-20.7)$ & 0.016 \\
\hline Lactate dehydrogenase at hospitalization (U/L) & $272(230-322)$ & $295(230-332)$ & $260(229-321.5)$ & 0.399 \\
\hline $\begin{array}{l}\text { Lactate dehydrogenase at conjunctival swab } \\
(U / L)\end{array}$ & $256(215-287)$ & $256(217-311)$ & $255.5(210-278.5)$ & 0.817 \\
\hline Total lymphocytes at hospitalization $\left(10^{9} \mathrm{cell} / \mathrm{s} / \mathrm{L}\right)$ & $1(0.7-1.3)$ & $0.9(0.8-1.1)$ & $1.1(0.7-1.3)$ & 0.493 \\
\hline Total lymphocytes at conjunctival swab & $1.2(0.8-1.6)$ & $1.3(1-1.6)$ & $1(0.8-1.5)$ & 0.438 \\
\hline
\end{tabular}


Table 1 Patients' characteristics according to the presence of ocular symptoms (Continued)

\begin{tabular}{|c|c|c|c|c|}
\hline Variable & $\begin{array}{l}\text { Overall } \\
(n=46)\end{array}$ & $\begin{array}{l}\text { With ocular symptoms or } \\
\text { signs } \\
(n=17)\end{array}$ & $\begin{array}{l}\text { Without ocular symptoms or } \\
\text { signs } \\
(n=29)\end{array}$ & $\begin{array}{l}p- \\
\text { value }^{\S}\end{array}$ \\
\hline Total monocytes at hospitalization $\left(10^{9} \mathrm{cell} / \mathrm{s} / \mathrm{L}\right)$ & $0.45(0.3-0.7)$ & $0.5(0.3-0.7)$ & $0.4(0.3-0.6)$ & 0.527 \\
\hline $\begin{array}{l}\text { Total monocytes at conjunctival swab }\left(10^{9} \text { cells/ }\right. \\
\text { L) }\end{array}$ & $0.5(0.4-0.7)$ & $0.5(0.5-0.8)$ & $0.4(0.3-0.7)$ & 0.081 \\
\hline Total neutrophils at hospitalization $\left(10^{9} \mathrm{cell} / \mathrm{s} / \mathrm{L}\right)$ & $4.2(2.5-6.2)$ & $5.2(3.4-9.5)$ & $4(2.4-5.7)$ & 0.227 \\
\hline $\begin{array}{l}\text { Total neutrophils at conjunctival swab }\left(10^{9} \text { cells/ }\right. \\
\text { L) }\end{array}$ & $4.55(2.5-5.6)$ & $5(4-5.6)$ & $4(2.3-5.5)$ & 0.158 \\
\hline PCR at hospitalization (mg/L) & $39.05(19-72.1)$ & $65.1(30.4-80.9)$ & $31.1(14-62)$ & 0.041 \\
\hline PCR at conjunctival swab (mg/L) & $18(5.4-38.4)$ & $29.6(10.6-38.4)$ & $11.05(3.45-36.2)$ & 0.228 \\
\hline PCT at hospitalization (ng/mL) & $0.91(0.35-2.06)$ & $0.67(0.35-2.06)$ & $1.14(0.56-1.61)$ & 0.999 \\
\hline PCT at conjunctival swab (ng/mL) & $0.65(0.39-1.01)$ & $0.95(0.43-1.14)$ & $0.64(0.35-0.85)$ & 0.368 \\
\hline Platelet at hospitalization $\left(10^{9} / \mathrm{L}\right)$ & $188.5(150-225)$ & $204(169-225)$ & $176(147-215)$ & 0.195 \\
\hline Platelet at conjunctival swab $\left(10^{9} / \mathrm{L}\right)$ & $193(159-258)$ & $191(171-258)$ & $195(154-254)$ & 0.741 \\
\hline RDW at hospitalization (\%) & $13.9(12.7-15.3)$ & $15.1(13.7-16.5)$ & $13.2(12.5-14.4)$ & 0.012 \\
\hline RDW at conjunctival swab (\%) & $14.1(12.6-15.5)$ & $15(14.3-16.7)$ & $13.2(12.4-14.4)$ & 0.001 \\
\hline White blood cells at hospitalization ( $10^{9}$ cells/L) & $5.85(4.1-7.7)$ & $6.5(4.7-10.4)$ & $5.5(3.8-7.3)$ & 0.274 \\
\hline $\begin{array}{l}\text { White blood cells at conjunctival swab } \\
\left(10^{9} \mathrm{cell} / \mathrm{L} / \mathrm{L}\right)\end{array}$ & $6.2(4.3-7.9)$ & $7.3(5.5-8)$ & $5.3(4.1-7.7)$ & 0.106 \\
\hline D-Dimer at hospitalization ( $\mu \mathrm{g} / \mathrm{mL})$ & $0.64(0.43-1.27)$ & $0.65(0.59-1.53)$ & $0.6(0.38-1.01)$ & 0.235 \\
\hline D-Dimer at conjunctival swab $(\mu \mathrm{g} / \mathrm{mL})$ & $0.4(0.33-0.63)$ & $0.58(0.34-0.71)$ & $0.38(0.27-0.55)$ & 0.165 \\
\hline $\mathrm{PaO}_{2} / \mathrm{FiO}_{2}$ at hospitalization (\%) $(n=28)$ & $3.04(2.05-3.36)$ & $2.83(1.77-3.32)$ & $3.22(2.07-3.42)$ & 0.416 \\
\hline $\mathrm{PaO}_{2} / \mathrm{FiO}_{2}$ at conjunctival swab (\%) $(n=14)$ & $2.36(1.61-3.18)$ & $2.04(1.84-2.34)$ & $2.75(1.41-3.4)$ & 0.533 \\
\hline $\mathrm{SaO}_{2}$ at hospitalization (\%) $(n=40)$ & $94.55(92.4-96.15)$ & $94.4(91.8-96.3)$ & $94.9(92.4-96)$ & 0.459 \\
\hline $\mathrm{SaO}_{2}$ at conjunctival swab (\%) $(n=23)$ & $95.7(94.2-97.3)$ & $97.4(96.3-99.3)$ & $95.3(94.2-96)$ & 0.069 \\
\hline Uric acid at hospitalization (mg/dL) & $4.4(3.65-6.35)$ & $3.9(3.8-6.3)$ & $4.5(3.6-6.6)$ & 0.630 \\
\hline Uric acid at conjunctival swab (mg/dL) & $4.3(3.7-6)$ & $3.9(3.8-5.2)$ & $4.65(3.5-6.2)$ & 0.467 \\
\hline Urea at hospitalization (mg/dL) & $32(23.5-42.5)$ & $33(29-49)$ & $31(23-40)$ & 0.254 \\
\hline Urea at conjunctival swab (mg/dL) & $31(24-46)$ & $39(31-49)$ & $27(22-40)$ & 0.189 \\
\hline ALT at hospitalization (U/L) & $31.5(18-39.5)$ & $31(15-36)$ & $32(21-41)$ & 0.244 \\
\hline ALT at conjunctival swab (U/L) & $33.5(20-52.5)$ & $32.5(22-52.5)$ & $35(18.5-55)$ & 0.678 \\
\hline AST at hospitalization (U/L) & $27(23-36)$ & $33(21-46)$ & $26(23.5-32)$ & 0.331 \\
\hline AST at conjunctival swab (U/L) & $27(22-36)$ & $27(23.5-34.5)$ & $27(21-47)$ & 0.742 \\
\hline CK at hospitalization (U/L) & $65.5(46-140)$ & $131(37-249)$ & $62(46-100)$ & 0.227 \\
\hline CK at conjunctival swab (U/L) & $43(27-100)$ & $27(22-49)$ & $56(35.5-100)$ & 0.056 \\
\hline Creatinine at hospitalization (mg/dL) & $0.96(0.77-1.1)$ & $0.95(0.77-1.28)$ & $0.96(0.77-1.07)$ & 0.594 \\
\hline Creatinine at conjunctival swab (mg/dL) & $0.83(0.73-1.01)$ & $0.88(0.79-1)$ & $0.78(0.72-1.03)$ & 0.400 \\
\hline Nasopharyngeal swab Ct at conjunctival swab & $\begin{array}{l}30.38(25.05- \\
32.75)\end{array}$ & $29.5(24.34-32.75)$ & $32.51(25.05-35.01)$ & 0.432 \\
\hline
\end{tabular}

Abbreviations: PCR, polymerase chain reaction; PCT, procalcitonin; RDW, red cell distribution width; ALT, alanine transaminase; AST, aspartate aminotransferase; CK, creatine kinase

Results are described by median (IQR) or frequency (\%), as appropriate

$\S$ by chi-square/Fisher's exact test (categorical variables) or Wilcoxon rank-sum test (continuous variables)

$49.4)$ vs. $8 \mathrm{pg} / \mathrm{ml}(3.6-20.7), p=0.02$. Red cell distribution width was also significantly higher: $15(14.3-16.7)$ vs 13.2 (12.4-14.4), $p=0.001$. Finally, oxygen saturation, $\mathrm{PaO} 2 /$ $\mathrm{FiO} 2$ ratio or the number of patients treated with nasal cannula or Venturi mask or non-invasive mechanical ventilation were not affected by the presence of ocular signs or symptoms. Detailed information about patients presenting ocular signs or symptoms is reported in Table 2. 


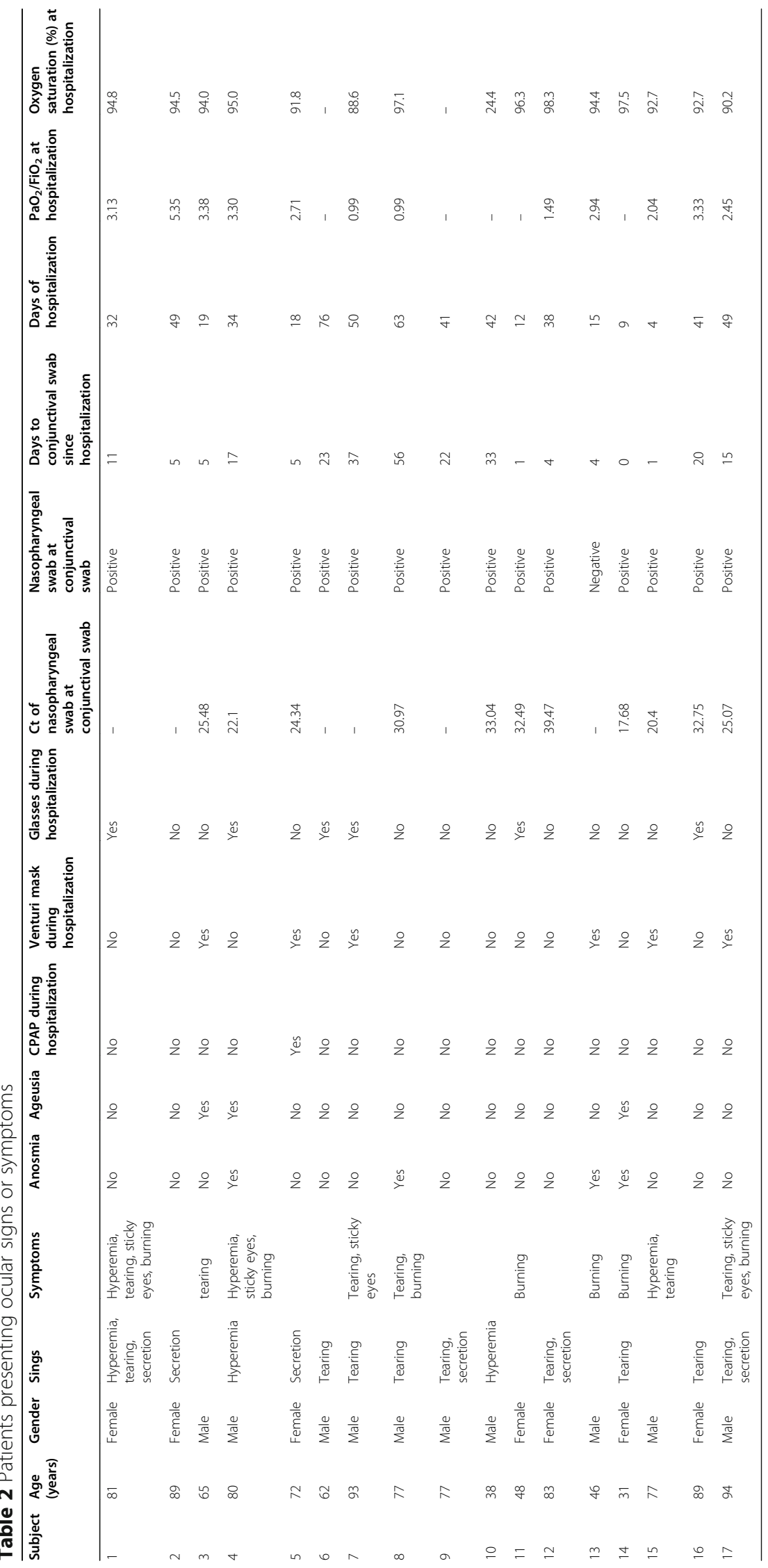


At multivariate analysis, plasma concentrations of Interleukin-6 and red cell distribution width were on average higher in patients with signs or symptoms (IL-6 $\beta:+32.6,95 \% \mathrm{CI}: 1.33-64.0, p=0.042$; RDW $\beta:+2.15$, 95\%CI: $0.91-3.39, p=0.001)$ compared to patients without them after adjusting for confounders; a marginally significant difference was seen in $\mathrm{Ct}$ values suggesting lower values (i.e. higher viral load) for patients with signs or symptoms when adjusting for confounders ( $\mathrm{Ct} \beta$ : -4.32 , 95\% CI: $-9.30,0.66, p=0.086$ ).

\section{Discussion}

In this paper, we show that conjunctivitis symptoms/ signs are associated with COVID-19 disease course. This is supported by a number of observations. First, the finding of elevated levels of IL-6, an important predictor of disease severity [10] [11]. Second, patients with ocular manifestations showed lower $\mathrm{Ct}$ values (i.e. higher viral load) in rhinopharyngeal swabs. Third, we also found that patients with ocular symptoms had a longer hospitalization, suggesting a relationship between ocular symptoms and severity of the disease. However, no significant difference in patients' age -one of the most important prognostic factor-was found between the two groups. Similarly, no significant difference was detected in the type of therapies administered during the hospital stay, which could have had an impact on viral clearance. Importantly, ocular signs/symptoms were not induced by ventilatory support, as our analysis failed to detect any association between the two.

Therefore, our study suggests that testing for ocular symptoms/signs could be a helpful tool to alert clinicians on a more severe clinical phenotype. In this vein, our results are corroborated by findings from Ping Wu et al. [12]

We did not find expression of COVID-19 RNA on the conjunctiva of our patients, similarly to others, who repeatedly tested a cohort of COVID-19 patients [13]. Azzolini et al [14] recently found virus expression in the conjunctiva, although clinical and methodological differences make comparisons difficult. However, the role of the ocular mucosa as a potential entry site or as a potential reservoir for the virus remains unclear.

We acknowledge some limitations including the crosssectional design of the study, the limited number of patients and the timing of swab collection that varied as a consequence of the critical situation induced by COVID-19 in our hospital.

Importantly, ocular signs/symptoms were not induced by ventilatory support, as our statistical analysis failed to detect any association between the two.

\section{Conclusion}

In summary, we found that over a third of the patients had ocular signs or symptoms and about a quarter presented anosmia and ageusia. While additional studies are needed to confirm our findings, we propose that testing ocular signs and symptoms at hospitalization can be an effective, non-invasive and rapid screening measure of COVID-19 patients.

\section{Abbreviations \\ SARS-CoV-2: Severe acute respiratory syndrome coronavirus 2; Ct: cycle threshold; $\mathrm{Cl}$ : confidence interval}

\section{Authors' contributions}

MR, EB collected the samples, completed the database and contributed to manuscript writing; LG performed statistical analysis, critically revised data and contribute to manuscript writing; AC contributed to manuscript writing and critically revised data; GF contributed to sample collection, wrote the manuscript and critically revised the data. The author(s) read and approved the final manuscript.

\section{Funding}

No funding has been required for this study.

Availability of data and materials

The datasets used and/or analysed during the current study are available from the corresponding author on reasonable request.

\section{Declarations}

Ethics approval and consent to participate

The study was conducted in accordance with the Declaration of Helsinki8 and the evaluated patients are part of the COVID-19 institutional clinicalbiological cohort (Covid-BioB registered on the ClinicalTrials.gov website: NCT04318366), whose study protocol was approved by the Hospital Ethics Committee (protocol number 34/int/2020). Informed consent was obtained according to the Ethic Committee guidelines.

\section{Consent for publication}

Does not apply.

\section{Competing interests}

None.

\section{Author details}

${ }^{1}$ Infectious Diseases, IRCCS San Raffaele Scientific Institute, Milan, Italy. ${ }^{2}$ Vita-Salute San Raffaele University, Milan, Italy. ${ }^{3}$ Cornea and Ocular Surface Disease Unit, Eye Repair Lab, IRCCS San Raffaele Scientific Institute, Via Olgettina, 60, 20132 Milan, Italy.

Received: 24 May 2021 Accepted: 5 September 2021

Published online: 22 September 2021

\section{References}

1. Nasiri N, Sharifi H, Bazrafshan A, Noori A, Karamouzian M, Sharifi A (2021) Ocular Manifestations of COVID-19: A Systematic Review and Meta-analysis. J Ophthalmic Vis Res 16(1):103-112. https://doi.org/10.18502/jovr.v16i1.8256

2. Sen M, Honavar SG, Sharma N, Sachdev MS (2021) COVID-19 and eye: a review of ophthalmic manifestations of COVID-19. Indian J Ophthalmol 69(3):488-509. https://doi.org/10.4103/ijo. IJO_297_21

3. Scalinci SZ, Trovato BE (2020) Conjunctivitis can be the only presenting sign and symptom of COVID-19. IDCases. 20:e00774. https://doi.org/10.1016/j. idcr.2020.e00774

4. Chen L, Deng C, Chen X, et al. (2020) Ocular manifestations and clinical characteristics of 535 cases of COVID-19 in Wuhan, China: a cross-sectional study. Acta Ophthalmol;98(8). doi:https://doi.org/10.1111/aos.14472

5. De Cobelli F, Palumbo D, Ciceri F et al (2021) Pulmonary vascular thrombosis in COVID-19 pneumonia. J Cardiothorac Vasc Anesth Published online January 13. https://doi.org/10.1053/j.jvca.2021.01.011

6. Mancini N, Clementi N, Ferrarese R, Ambrosi A, Tonelli M, Zangrillo A, Landoni G, Clementi M (2021) Very high SARS-CoV-2 load at the emergency department presentation strongly predicts the risk of admission to the 
intensive care unit and death. Clin Chem Lab Med Published online January 21(6):e247-e250. https://doi.org/10.1515/cclm-2020-1709

7. Tom MR, Mina MJ (2020) To interpret the SARS-CoV-2 test, consider the cycle threshold value. Clin Infect Dis 71(16):2252-2254. https://doi.org/10.1 093/cid/ciaa619

8. World Medical Association Declaration of Helsinki (2013) JAMA. 310(20):2191. doi:https://doi.org/10.1001/jama.2013.281053

9. Agresti A, Coull BA (1998) Approximate is better than "exact" for interval estimation of binomial proportions. Am Stat 52(2):119-126. https://doi.org/1 0.1080/00031305.1998.10480550

10. Udomsinprasert W, Jittikoon J, Sangroongruangsri S, Chaikledkaew U (2021) Circulating levels of Interleukin-6 and Interleukin-10, but not tumor necrosis factor-alpha, as potential biomarkers of severity and mortality for COVID-19: systematic review with Meta-analysis. J Clin Immunol 41(1):11-22. https:// doi.org/10.1007/s10875-020-00899-z

11. Cavalli G, Larcher A, Tomelleri A, Campochiaro C, Della-Torre E, de Luca G, Farina N, Boffini N, Ruggeri A, Poli A, Scarpellini P, Rovere-Querini P, Tresoldi M, Salonia A, Montorsi F, Landoni G, Castagna A, Ciceri F, Zangrillo A, Dagna L (2021) Interleukin-1 and interleukin-6 inhibition compared with standard management in patients with COVID-19 and hyperinflammation: a cohort study. Lancet Rheumatol 3(4):e253-e261. https://doi.org/10.1016/S2665-9913 (21)00012-6

12. Wu P, Duan F, Luo C, Liu Q, Qu X, Liang L, Wu K (2020) Characteristics of ocular findings of patients with coronavirus disease 2019 (COVID-19) in Hubei Province. China JAMA Ophthalmol 138(5):575-578. https://doi.org/10.1 001/jamaophthalmol.2020.1291

13. Seah IYJ, Anderson DE, Kang AEZ, Wang L, Rao P, Young BE, Lye DC, Agrawal R (2020) Assessing viral shedding and infectivity of tears in coronavirus disease 2019 (COVID-19) patients. Ophthalmology. 127(7):977979. https://doi.org/10.1016/j.ophtha.2020.03.026

14. Azzolini C, Donati S, Premi E, Baj A, Siracusa C, Genoni A, Grossi PA, Azzi L, Sessa F, Dentali F, Severgnini P, Minoja G, Cabrini L, Chiaravalli M, Veronesi G, Carcano G, Maffioli LS, Tagliabue A (2021) SARS-CoV-2 on ocular surfaces in a cohort of patients with COVID-19 from the Lombardy region. Italy JAMA Ophthalmol Published online March 4. https://doi.org/10.1001/jama ophthalmol.2020.5464

\section{Publisher's Note}

Springer Nature remains neutral with regard to jurisdictional claims in published maps and institutional affiliations.

\section{Submit your manuscript to a SpringerOpen ${ }^{\circ}$ journal and benefit from:}

- Convenient online submission

- Rigorous peer review

- Open access: articles freely available online

- High visibility within the field

- Retaining the copyright to your article

Submit your next manuscript at $\boldsymbol{\nabla}$ springeropen.com 